\title{
MISTURA DE ÁCIDO PIROLENHOSO COM GLIFOSATO NO CONTROLE DE Cynodon dactylon (L.) Pers.
}

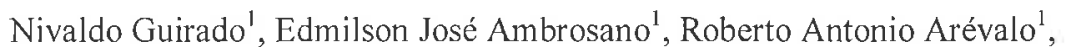
Paulo Cesar Doimo Mendes' ${ }^{1}$ Fabricio Rossi ${ }^{1}$

\section{RESUMO}

A matospecie Cynodon dactylon (L.) Pers. conhecida no Brasil como grama-seda é considerada infestante importante em diversas culturas. O herbicida mais utilizado para seu controle é o glifosato aplicado em pósemergência. O uso indiscriminado de herbicidas tem levado a sérios problemas, desde a intoxicação do trabalhador rural até a resistência das plantas ao seu uso. O objetivo deste trabalho foi reduzir a dose de glifosato aplicada, misturando a este, ácido pirolenhoso para atuar como elicitor. O experimento foi realizado em vasos de $35 \mathrm{~L}$ de capacidade, com 0,16 metros quadrados de área, sendo 8 tratamentos repetidos 4 vezes em blocos ao acaso. Os tratamentos e doses do produto comercial utilizado foram: glifosato 6,0; 4,5 e 3,0 $\mathrm{L} \mathrm{ha}^{-1}$; ácido pirolenhoso destilado (BioBIRE) 1,5 e 3,0 $\mathrm{L} \mathrm{ha}^{-1}$ e as misturas glifosato 4,5 $\mathrm{L} \mathrm{ha}^{-1}+$ BioBIRE 1,5 L ha ${ }^{-1}$; glifosato 3,0 $\mathrm{L} \mathrm{ha}^{-1}+$ BioBIRE 3,0 $\mathrm{L} \mathrm{ha}^{-1}$ e testemunha. A quantidade aplicada foi correspondente a um volume de calda de $300 \mathrm{~L} \mathrm{ha}^{-1}$. A avaliação foi realizada 30 dias após a aplicação dos produtos, verificando-se o peso da fitomassa seca. Os melhores efeitos foram obtidos com glifosato $4,5 \mathrm{~L} \mathrm{ha}^{-1}+$ BioBIRE 1,5 L ha-1, seguido por glifosato 6,0 e 4,5 $\mathrm{L} \mathrm{ha}^{-1}$ e glifosato $3,0 \mathrm{~L}$ $\mathrm{ha}^{-1}+$ BioBIRE 3,0 $\mathrm{L} \mathrm{ha}^{-1}$. Contudo, tais tratamentos não diferiram significativamente entre si. O ácido pirolenhoso na dosagem de $0,5 \%$

${ }^{1}$ Apta/Pólo Regional de Desenvolvimento Tecnológico dos Agronegócios do Centro Sul, Piracicaba, SP. E-mail: nguiradoraptaregional.sp.goy.br. 
estimulou e a 1,0\% inibiu o desenvolvimento das plantas sendo nesta, o peso da fitomassa seca inferior ao da testemunha.

Palavras-chave: manejo químico, CYNDA, herbicida, grama-seda.

\title{
PYROLIGNEOUS ACID MIXED WITH GLYPHOSATE FOR Cynodon dactylon (L.) Pers CONTROL.
}

\begin{abstract}
The weed Cynodon dactylon (L.) Pers. known in Brazil as
\end{abstract} bermudagrass is considered as a serious carrier of diseases in several crops. The herbicide glyphosate is one of the best and most used one for postemergence application for bermudagrass control. Indiscriminate use of herbicides has led to serious problems, from rural worker intoxication to plants resistance. The aim of this work was to reduce the herbicide effect by including pyroligneous acid. The experiment was done in 35 litres vases, in an area of 0.16 square meters, with 8 treatments replicated 4 times in randomized blocks. The treatments and doses of the commercial product used were: glyphosate, 6.0, 4.5 and 3.0 $\mathrm{L} \mathrm{ha}^{-1}$; pyroligneous acid distillery (BioBIRE) 1.5 and $3.0 \mathrm{~L} \mathrm{ha}^{-1}$; glyphosate mixtures $4.5 \mathrm{~L} \mathrm{ha}^{-1}+$ BioBIRE 1.5 $\mathrm{L} \mathrm{ha}^{-1}$; glyphosate 3.0 L ha ${ }^{-1}+$ BioBIRE $3.0 \mathrm{~L} \mathrm{ha}^{-1}$ and control. The amount applied corresponded to a volume of $300 \mathrm{~L} \mathrm{ha}^{-1}$. The evaluation was done 30 days after the application, by verifying the dry phytomass weight. The best effects were obtained with glyphosate $4.5 \mathrm{Lha}^{-1}+$ BioBIRE 1.5 $\mathrm{Lha}^{-1}$, followed by glyphosate, 6.0 and $4.5 \mathrm{Lha}^{-1}$ and glyphosate 3.0 $\mathrm{Lha}^{-1}+$ BioBIRE 3.0 Lha $^{-1}$. However, the treatments did not differ significantly. The pyroligneous acid in $0.5 \%$ doses stimulated the plants development and in the $1.0 \%$ doses inhibited leaving the dry phytomass lighter than control.

Key words: Chemical management, $C Y N D A$, herbicide, bermudagrass. 


\section{INTRODUÇÃO}

A matospecie CYNDA, Cynodon dactylon (L.) Pers. conhecida no Brasil como grama-seda, capim de burro e grama rasteira entre outros, tem ampla distribuição mundial. É considerada infestante importante nas culturas: de algodão, amendoim, café, citros, fumo, além de muitas outras. Em cana-de-açúcar, uma alta infestação pode reduzir em até $80 \%$ a produção, além de reduzir o número de cortes e a vida útil do canavial (Kissmann, 1997). A planta compete desde os primeiros 30 dias até o desenvolvimento completo da cultura da cana, sendo que em áreas infestadas o desenvolvimento fica comprometido (Arévalo, 1992).

CYNDA é uma infestante de difícil controle e ou erradicação. A sua disseminação ocorre pelo cultivo mecânico, maquinarias agrícolas, animais, água e vento. Reproduz-se por sementes e se multiplica por estolões e rizomas (Lorenzi, 2000).

Dos 104 herbicidas registrados para a agricultura brasileira (Lorenzi, 2000) relata que um dos melhores e mais utilizado para aplicação em pós-emergência é o glifosato. Labrada (1996) considera o glifosato aplicado em pós-emergência como um herbicida efetivo para controle de CYNDA em pomares.

O mercado brasileiro de defensivos agrícolas tem sido bastante dinâmico, tendo aumentado o consumo de US\$ 1 bilhão em 1990, para US\$ 2,3 bilhões em 1999, sendo os herbicidas o principal segmento de mercado com cerca de 50\%. Em termos de variação de consumo, os herbicidas tiveram um aumento de 113\% de acordo com Martinelli e Waquil (2001). A ampla variabilidade genética é uma das principais características das plantas daninhas, que permite a adaptação e a sobrevivência dessas espécies em diversas condiçôes ambientais e do agroecossistema. $\mathrm{O}$ uso indiscriminado dos herbicidas tem levado a sérios problemas, desde a intoxicação do trabalhador rural até a resistência das plantas ao seu uso. Devido à utilização 
intensiva de herbicidas nas últimas décadas, algumas populações de plantas daninhas foram selecionadas em resposta ao distúrbio ambiental provocado pela pressão de seleção dos herbicidas, com a seleção de biótipos a eles resistentes (Christoffoleti, 2003). A ocorrência de alta densidade de plantas daninhas resistentes aos herbicidas pode limitar a produção das culturas agrícolas. Portanto, torna-se necessário o desenvolvimento de técnicas agrícolas que minimizem a utilização destes produtos. Segundo Roman et al. (2004), a eficácia de herbicidas é influenciada por diversos fatores que afetam a absorção e a translocação desses compostos na planta. A potencialização, do efeito do herbicida com redução da dose aplicada, seria extremamente útil neste sentido. $O$ ácido pirolenhoso é utilizado na agricultura como estimulante do crescimento de plantas, o que pode vir a favorecer uma maior absorção de substâncias aplicadas e com isso aumentar a sua influência sobre as plantas.

O ácido pirolenhoso ou licor pirolenhoso é resultante da condensação da fumaça obtida durante a incineração da madeira de diversas espécies de plantas. É constituído de cerca de 200 diferentes compostos orgânicos, com predomínio do ácido acético (Myasaka et al., 2001). Além do ácido acético, também são encontrados o ácido propiônico, metanol, maltol, guaiacol e cresol. O ácido pirolenhoso vem sendo utilizado como fertilizante orgânico (Esechie et al., 1998; Shibayama et al., 1998; Tsuzuki et al., 2000), desinfectante de solo (Doran, 1932) repelente de pragas de insetos, regulador de $\mathrm{pH}$ de soluções, acelerador de produção de compostos orgânicos, na prevenção algumas doenças de plantas cultivadas (Miyasaka et al., 2001), além de estimulante do crescimento de plantas (Tsuzuki et al., 1989; Uddin et al., 1995; Du et al., 1997 e 1998). Devido ao efeito estimulante do ácido pirolenhoso e sua fácil absorção pelas plantas o presente trabalho foi realizado visando sua utilização na redução da dose do herbicida glifosato no controle de $C Y N D A$. 


\section{MATERIAL E MÉTODOS}

O experimento foi implantado na primavera de 2004, no Pólo Regional Centro Sul, em Piracicaba-SP, em vasos de 35 L de capacidade, com 0,16 metros quadrados de área, os quais foram preenchidos com 1 parte de solo (Argissolo Vermelho Amarelo distrófico), 1 parte de húmus de minhoca e 1 parte de areia, onde foram plantadas 5 segmentos unigemares de estolões de CYNDA. A cada 2 dias, essa mistura era irrigada com regador, gastando-se 2,0 L de água por vaso. Trinta dias após a semeadura procedeu-se a aplicação dos produtos sobre as mesmas. O experimento foi formado por 8 tratamentos repetidos 4 vezes em blocos ao acaso. Foram utilizados os produtos glifosato 360 g. $\mathrm{L}^{-1}$ i.a. e ácido pirolenhoso destilado (BioBIRE). Os tratamentos e doses do produto comercial foram: glifosato 6,0 L ha ${ }^{-1}$; glifosato 4,5 $\mathrm{L} \mathrm{ha}^{-1}$; BioBIRE 1,5 L ha ${ }^{-1}$; glifosato 4,5 $\mathrm{L} \mathrm{ha}^{-1}+$ BioBIRE 1,5 L ha' ${ }^{-1}$; glifosato 3,0 $\mathrm{L} \mathrm{ha}^{-1}$; BioBIRE 3,0 L ha ${ }^{-1}$; glifosato 3,0 L $\mathrm{ha}^{-1}+$ BioBIRE 3,0 L ha ${ }^{-1}$ e Testemunha. A aplicação dos produtos foi através de pulverização gastando-se $300 \mathrm{~L} \mathrm{ha}^{-1}$. Trinta dias após a aplicação, coletou-se a $C Y N D A$ e verificou-se o peso da fitomassa seca. Procedeu-se à análise da variância (ANOVA) e foram realizados testes de comparação de médias Duncan $(\alpha=0,05)$.

\section{RESULTADOS E DISCUSSÃO}

Através da análise do peso da fitomassa seca de $C Y N D A$, foi verificado que o ácido pirolenhoso BioBIRE aplicado na dose de 0,5\% proporcionou aumento de peso désta, e na dose de 1,0\% promoveu inibição, sendo o peso da fitomassa seca inferior ao da testemunha (Tabela 1). $O$ ácido pirolenhoso 1,5 $\mathrm{L} \mathrm{ha}^{-1} \mathrm{em}$ mistura com o herbicida glifosato 4,5 $\mathrm{L} \mathrm{ha}^{-1}$, proporcionou menor peso de fitomassa seca de $C Y N D A$ comparando com os demais tratamentos e, conseqüentemente, foi o melhor controle. Este tratamento embora mais eficiente que os demais, não diferiu 
estatisticamente da utilização de glifosato 6,0 e 4,5 $\mathrm{L} \mathrm{ha}^{-1}$ e glifosato $3,0 \mathrm{~L}$ $\mathrm{ha}^{-1}$ mais ácido pirolenhoso a $3,0 \mathrm{~L} \mathrm{ha}^{-1}$.

Tabela 1 - Peso da fitomassa seca (g) aos 30 dias após a aplicação dos tratamentos.

\section{TRATAMENTOS}

\begin{tabular}{|c|c|c|}
\hline & \multicolumn{2}{|c|}{$-g-$} \\
\hline BioBIRE $1,5 \mathrm{~L} \mathrm{ha}^{-1}$ & 179,18 & A \\
\hline Testemunha & 141,66 & B \\
\hline BioBIRE 3,0 $\mathrm{L} \mathrm{ha}^{-1}$ & 111,21 & $\mathrm{C}$ \\
\hline Glifosato $3,0 \mathrm{~L} \mathrm{ha}^{-1}$ & 69,32 & $\mathrm{D}$ \\
\hline Glifosato 3,0 $\mathrm{L} \mathrm{ha}^{-1}+$ BioBIRE 3,0 $\mathrm{L} \mathrm{ha}^{-1}$ & 61,70 & $\mathrm{DE}$ \\
\hline Glifosato $6,0 \mathrm{~L} \mathrm{ha}^{-1}$ & 59,25 & $\mathrm{DE}$ \\
\hline Glifosato $4,5 \mathrm{~L} \mathrm{ha}^{-1}$ & 57,33 & DE \\
\hline Glifosato 4,5 $\mathrm{L} \mathrm{ha}^{-1}+$ BioBIRE 1,5 $\mathrm{L} \mathrm{ha}^{-1}$ & 47,12 & $\mathrm{E}$ \\
\hline $\mathrm{CV}(\%)$ & 19,63 & \\
\hline
\end{tabular}

Médias seguidas de mesma letra não diferem entre si pelo teste de Duncan $(\mathrm{P}>0,05)$.

Os tratamentos no qual o glifosato foi utilizado sozinho na dose de 6,0 e 4,5 litros do produto comercial por hectare e glifosato $3,0 \mathrm{~L} \mathrm{ha}^{-1}$ juntamente com ácido pirolenhoso a $3,0 \mathrm{~L} \mathrm{ha}^{-1}$, não apresentaram diferenças significativas entre si, mas diferiram dos tratamentos com ácido pirolenhoso e da testemunha. Os resultados mostram também, que o ácido pirolenhoso utilizado sozinho na dose de $0,5 \%\left(1,5 \mathrm{~L} \mathrm{ha}^{-1}\right)$ foi o que promoveu maior desenvolvimento das plantas, diferindo de todos os demais tratamentos. Entretanto, o ácido pirolenhoso utilizado na dose de $1 \%\left(3,0 \mathrm{~L} \mathrm{ha}^{-1}\right)$ inibiu o desenvolvimento de $C Y N D A$, sendo o peso seco inferior ao tratamento testemunha. De acordo com a cultura em que se pulveriza este ácido tem que se estabelecer à dose ideal. Em tomate pulverizações na sementeira com dose de $1 \%$, acelera a germinação e desenvolvimento das plantas. Já em 
citros pulverização do ácido reduziu o desenvolvimento de porta-enxertos de limoeiro 'Cravo' com a dose de 5 e $10 \mathrm{~cm}^{3} \mathrm{dm}^{-3}$ (Zanetti et al. 2003).

\section{CONCLUSÕES}

Os melhores efeitos foram obtidos com glifosato $4,5 \mathrm{~L} \mathrm{ha}^{-1}+$ BioBIRE 1,5 $\mathrm{L} \mathrm{ha}^{-1}$, seguido por glifosato 6,0 e 4,5 $\mathrm{L} \mathrm{ha}^{-1}$ e glifosato $3,0 \mathrm{~L}$ $\mathrm{ha}^{-1}+$ BioBIRE 3,0 $\mathrm{L} \mathrm{ha}^{-1}$, os quais não diferiram entre si. O ácido pirolenhoso na dose de $0,5 \%$ estimulou o desenvolvimento da planta e a $1 \%$ inibiu.

\section{REFERÊNCIAS BIBLIOGRÁFICAS}

ARÉVALO, R.A. Manejo de las peores matospecies (malezas) de Saccharum spp. (caña de azúcar). In: SIMPOSIUM INTERNACIONAL MANEJO DE LA MALEZA: SITUACIÓN ACTUAL E PERSPECTIVAS, 1., Chapingo, México, 1992. Universidad Autónoma Chapingo. Memorias-UACH, p. 156.

CHRISTOFFOLETI, P.J. \& LOPEZ-OVEJERO, R. Principais aspectos da resistência de plantas daninhas ao herbicida glyphosate. Planta Daninha, 2003, v. 21, n. 3, p. 507-515.

HOLM, L.G.; PLUCKNETT, D.L.; PANCHO, J.V. \& HERBERGER, J.P. The world's worst weeds. Distribution and biology. Honolulu. The East-West Center by the University Press of Hawaii, 1977, p.25.

DORAN, W.L. Acetic acid and pyroligneous acid in comparision with formaldehyde as soil desinfectants. Journal of Agriculture Research, Washington, v.44, n.7, p.571-578, 1932. 
DU, H.G.; MORI, E.; TERAO, H.; TSUZUKI, E. Effect of the mixture of charcoal with pyrolegneous acid on shoot and root growth of sweet potate (Ipomoae batatas). Japanese Journal of Crop Science, BankyoKu, Tokio, v.67, n.2, p.149-152, 1998.

DU, H.G.; OGAWA, M.; ANDO, S.; TSUZUKI, E.; MURAYAMA, S. Effect of mixture of charcoal with pyrolegneous acid on sucorose content in netted melon (Cucumis melo L. var. reticulates Naud.) fruit. Japanese Journal of Crop Science, Bankyo-Ku, Tokio, v.66, n.3, p.369-373, 1997.

ESECHIE, H.A.; DHALIWAL, G.S.; ARORA, R.; RANDHAWA, N.S.; DHAWAN, A.K. Assessment of pyrolegneous liquid as a potential organic fertilizer. In: Ecological agriculture and sustainable development, 1997, Chandigarh, India. Proceedings...Chandigarh: Center for Research in Rural and Industrial Development, 1998. v.1, p.591-595.

KISSMANN, K.G. Plantas infestantes e nocivas - Tomo I, São Paulo, SP BASF $2^{a}$ ed., 1997, 825p.

LABRADA, R. Cynodon dactylon (L.) Pers. In: LABRADA, R. CASELEY, J. C. \& PARKER, C. Estudio FAO: Producción y Protección Vegetal, n. 120,1996, p. 50.

LORENZI, H. Plantas daninhas do Brasil. Terrestres, aquáticas, parasitas e tóxicas, $3^{\mathrm{a}}$ ed. Nova Odessa, SP, 2000, p.304.

MARTINELLI, O. \& WAQUIL, P.D. Comportamento tecnológico das empresas: O setor de defensivos agrícolas. Araraquara, SP, UNESP, 2001, 91p. Disponível em: http://www.finep.gov.br/estudos/arquivos/bibli_finep/ctc_defensivos_pdf. Acesso em: 23.02.2005. 
MYASAKA, S.; OHKAWARA, T.; NAGAI, K.; YAZAKI, H. \& SAKITA, M.N. Técnicas de produção e uso de fino de carvão e licor pirolenhoso. In: ENCONTRO DE PROCESSOS DE PROTEÇÃO DE PLANTAS CONTROLE ECOLÓGICO DE PRAGAS E DOENÇAS 1., Botucatu-SP. Agroecológica , 2001, p. 161-176.

ROMAN, E.S.; VARGAS, L.; RIBEIRO, M.C.F.; LUIZ, A.R.M. Influência do orvalho e volume de calda de aplicação do glyphosate na dessecação de Brachiaria plantaginea. Planta Daninha.Viçosa, MG. v.22, n.3, p.1-6, 2004.

SHIBAYAMA, H.; MASHIMA, K.; MITSUTOMI, M.; ARIMA, S. Effects of aplication of pyrolegneous acid solution produced in Karatsu city on growth and free sugar contents of storage roots of sweet potatoes. Marine and Higland Bioscience Center Report, Phukel, v.7, p.15-23, 1998.

TSUZUKI, E.; WAKIYAMA, Y.; ETO, H.; HANDA, H. Effect of pyrolegneous acid and mixture of charcoal with pyrolegneous acid on the growth and yield of rice plant. Japan Journal Crop Science, Bankyo-ku, Tokyo, v.58, n.4, p.592-597, 1989.

TSUZUKI, E.; MORIMITSU, T.; MATSUI, T. Effect of chemical compounds in pyrolegneous acid on root growth in rice plant. Japan Journal Crop Science, Bankyo-ku, Tokyo, v.66, n.4, p.15-16, 2000.

UDDIN, S.M.M.; MURAYAMA, S. ISHIMINE, Y.; TSUZUKI, E.; HARADA, J. Effect of mixture of charcoal with pyrolegneous acid on dry mather production and root growth of summer planted sugarcane (Saccharum officinarum L.). Japan Journal Crop Science, Bankyoku, Tokyo, v.64, n.4, p.747-753, 1995. 
ZANETTI, M.; CAZETTA, J.O.; MATTOS JÚNIOR, D.; CARVALHO, S.A. Uso de subprodutos de carvão vegetal na formação do portaenxerto limoeiro 'Cravo' em ambiente protegido. Revista Brasileira de Fruticultura, Jaboticabal, SP, v.25, n.3, p.508-512, 2003. 